\title{
Investigation on Synthesis, Growth, Structural and Optical Properties of a New Semiorganic Crystal 2-Aminopyridine Potassium Hydrogen Phthalate (2APKHP)
}

\author{
K. Arunadevi, R. Tamilselvi, D. Sivavishnu, R. Srineevasan, P. Ramadoss* \\ "Post Graduate and Research Department of Physics, Government Arts College, Tiruvannamalai-606603, \\ Tamilnadu, India. \\ "Corresponding author: dossrpg@yahoo.co.in
}

\begin{abstract}
A semiorganic 2-aminopyridine potassium hydrogen phthalate (2APKHP) crystal has been synthesized using slow evaporation solution growth technique at ambient temperature. The grown 2APKHP crystal was harvested with dimension $10 \times 18 \times 4 \mathrm{~mm}^{3}$. The lattice parameters of the as grown crystal are $a=6.49 \AA, b=9.63 \AA, c=13.27 \AA$ and volume $V=829 \AA^{3}$ were obtained by Single crystal $X$-ray diffraction analysis, reveals that the $2 A P K H P$ crystal belongs to orthorhombic crystal system with space group PCa2 ${ }_{1}$. The Powder X-ray diffraction analysis confirms the crystalline nature of $2 A P K H P$ crystal and the (hkl) values are indexed for corresponding intensity value using INDX software. The UV-vis-NIR transmission spectrum of $2 A P K H P$ in the range of 200-1000 $\mathrm{nm}$ shows lower cut-off wavelength was estimated from the optical studies. Nonlinear optical property of the powder sample 2APKHP was studied by Kurtz-Perry powder technique using Nd:YAG laser and the crystal exhibits second harmonic generation (SHG) property. Dielectric constant and dielectric loss of the sample have been measured for different frequencies at different temperatures.
\end{abstract}

Keywords: Solution growth techniques; XRD analysis; UV spectrum.

\section{Introduction}

In recent years, many significant achievements have occurred in the field of nonlinear optics because of the development of laser technology and new nonlinear optical materials of both inorganic and organic types [1]. An organic material shows poor mechanical and thermal properties and it is difficult to grow large optical quality crystal of these materials for device applications $[2,3]$. The organic materials have very large nonlinear susceptibilities compared with inorganic materials, but their use is foil by their low optical transparencies, poor mechanical properties, low laser damage threshold and an inability to produce and process large crystals. Pure inorganic nonlinear optical materials typically have excellent mechanical and thermal properties but posses relatively modest optical nonlinearities because of the lack of extended $\pi$-electron delocalization [4]. In view of these problem encountered with the organic materials, hybrid NLO materials, are explored as organic-inorganic complexes with stronger ionic bond and several single crystals were grown $[5,6]$. In this context several inorganic -organic crystals have been prepared, structurally elucidated and their physical properties were studied $[7,8]$. Semiorganic crystals have attracted many researcher because of their admirable combination of hyper-polarization, low angular sensitivity, excellent mechanical properties and their easiness to after their molecular structure in order to maximize the nonlinear properties [9-12]. Amino acid family crystals are of interest due to their attractive nonlinear optical properties [13, 14]. Monaco et.al, have reported the formation of 1:1 molecular compounds between amino acid and many different acids [15-19]. Potassium hydrogen phthalate crystals are playing an important role in the field of nonlinear optic materials, they are known SHG material that have long stability in devices due to their electro-optic properties and exhibit interesting in piezoelectric, pyroelectric and elastic properties that are useful in many applications [20-23]. Pyridine is a heterocyclic organic compound with the chemical formula $\mathrm{C}_{5} \mathrm{H}_{5} \mathrm{~N}$. Intensive studies on pyridine and pyridine derivatives have been made in the past, keeping in the view of their industrial importance both as fundamental building block and reagent in organic synthesis [24]. In this present investigation, we report on synthesis, growth, structure and optical properties of 2-aminopyridine potassium hydrogen phthalate (2APKHP) for the first time.

\subsection{Material synthesis of 2APKHP}

\section{Experimental Procedure}

A 2-aminopyridine potassium hydrogen phthalate (2APKHP) was synthesized in equimolar ratio 1:1 by using double distilled water as a solvent at room temperature. The chemical reaction of synthesized compound was shown below and the molecular scheme is shown in figure 1.

$$
\mathrm{C}_{5} \mathrm{H}_{6} \mathrm{~N}_{2}+\mathrm{C}_{8} \mathrm{H}_{5} \mathrm{KO}_{4} \rightarrow \mathrm{C}_{5} \mathrm{H}_{6} \mathrm{~N}_{2} \mathrm{C}_{8} \mathrm{H}_{5} \mathrm{KO}_{4}
$$

2 -aminopyridine + Potassium hydrogen phthalate $\rightarrow 2$ APKHP 
<smiles>[Y7]N(c1ccncc1)c1ccc(C(=O)O)c(C(=O)[O-])c1</smiles>

Figure 1. Molecular scheme of 2APKHP

The prepared mixture was stirred well for 8 hours using a magnetic stirrer to form a homogeneous solution. After filtration the solution was kept in an undisturbed place for slow evaporation. After 5 days the material was crystallized at bottom of the beaker. The material was recrystallized for purification and crystal growth.

\subsection{Growth process of 2 APKHP crystal}

The slow evaporation technique was used to grow single crystal of 2APKHP from aqueous solution. Optically good quality crystals, free from macro defects, obtained by self nucleation of the saturated solution, were used as seed crystals. Bulk size crystal of 2APKHP were grown using these seed crystals from saturated solution by slow evaporation method at room temperature. A good optical quality of crystal with dimension $10 \times 18 \times 4 \mathrm{~mm}^{3}$ was harvested in the period of 85 days. These crystals are non-hygroscopic and optically transparent. The photography of as grown crystal of 2APKHP was shown in figure 2 . The centroid and structural plane diagram of 2APKHP is shown in figure 3.

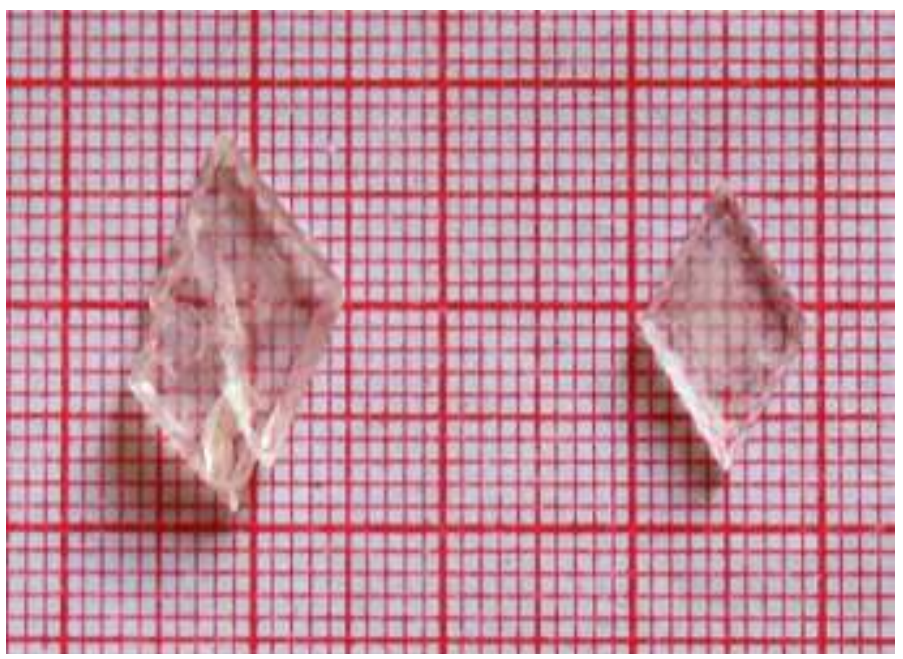

Figure 2. Photography of as grown crystal of 2APKHP

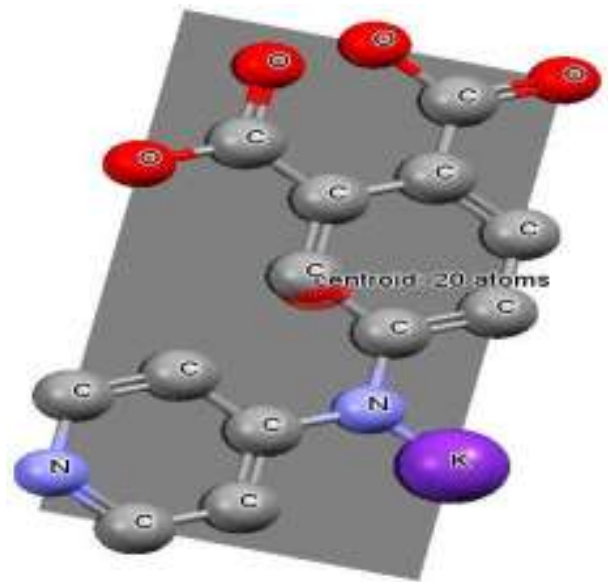

Figure 3. Centroid and Structural plane diagram of 2APKHP crystal 
Investigation on Synthesis, Growth, Structural and Optical Properties of a New Semiorganic ..

\subsection{Single crystal $X$-ray diffraction analysis}

\section{Results and Discussion}

Well shaped transparent single crystal of 2APKHP were selected and it was subjected to single crystal $\mathrm{X}$-ray diffraction analysis using ENRAF NONIUS CAD-4 automatic single crystal X-ray diffractrometer. This analysis reveals that the grown crystal 2APKHP crystallizes in orthorhombic crystal system with space group $\mathrm{Pca}_{1}$. The calculated lattice parameter for 2 APKHP crystal was found to be $\mathrm{a}=6.49 \AA, b=9.63 \AA, c=13.27 \AA$ and volume $\mathrm{V}=829 \AA^{3}$. Thus the XRD results confirms the incorporation of potassium hydrogen phthalate in 2-aminopyridine. From the single crystal XRD study of 2APKHP, there is a slight variation in lattice parameters and there is no change in crystal system in replacing 2-aminopyridine instead of thiourea in potassium hydrogen phthalate which is already reported [20]. From the table 1, the change of lattice parameter and structure indicates the presence of potassium hydrogen phthalate in 2-aminopyridine. The function groups determined by FT-IR analysis confirm the presence of metal ion, which is shown in table 2 .

Table 1 Lattice parameter and crystal system.

\begin{tabular}{|l|c|c|c|c|c|c|c|c|}
\hline \multicolumn{1}{|c|}{ Crystals } & $\mathbf{a}(\stackrel{\AA}{\mathbf{A}})$ & $\mathbf{b}(\stackrel{\AA}{\mathbf{A}})$ & $\mathbf{c}(\stackrel{\AA}{\mathbf{A}})$ & $\boldsymbol{\alpha}$ & $\boldsymbol{\beta}$ & $\boldsymbol{\gamma}$ & Volume $\left(\check{\AA}^{3}\right)$ & Crystal system \\
\hline $\begin{array}{l}\text { 2-amino } \\
\text { Pyridine [24] }\end{array}$ & 11.71 & 5.67 & 7.59 & $90^{\circ}$ & $95.6^{\circ}$ & $90^{\circ}$ & 504 & Monoclinic \\
\hline TKHP [20] & 6.43 & 9.56 & 13.24 & $90^{\circ}$ & $90^{\circ}$ & $90^{\circ}$ & 813 & Orthorhombic \\
\hline 2APKHP* & 6.49 & 9.63 & 13.27 & $90^{\circ}$ & $90^{\circ}$ & $90^{\circ}$ & 829 & Orthorhombic \\
\hline
\end{tabular}

*Present work

\subsection{Powder XRD analysis}

The powder sample of 2APKHP have been analyzed by using BRUCKER Germany (Model D8 advance) X-ray diffractrometer $\mathrm{CuK} \alpha\left(\lambda=1.5405 \mathrm{~A}^{\circ}\right.$.) radiation. The 2APKHP powder sample was scanned in the range of $0-80^{\circ}$ at the rate of $1^{\circ}$ per minute. The sharp and well defined Bragg's peaks at specific $2 \Theta$ angles confirm the good crystalline nature and purity of the grown crystal which is shown in figure 4 . The (hkl) values are fixed using INDX software.

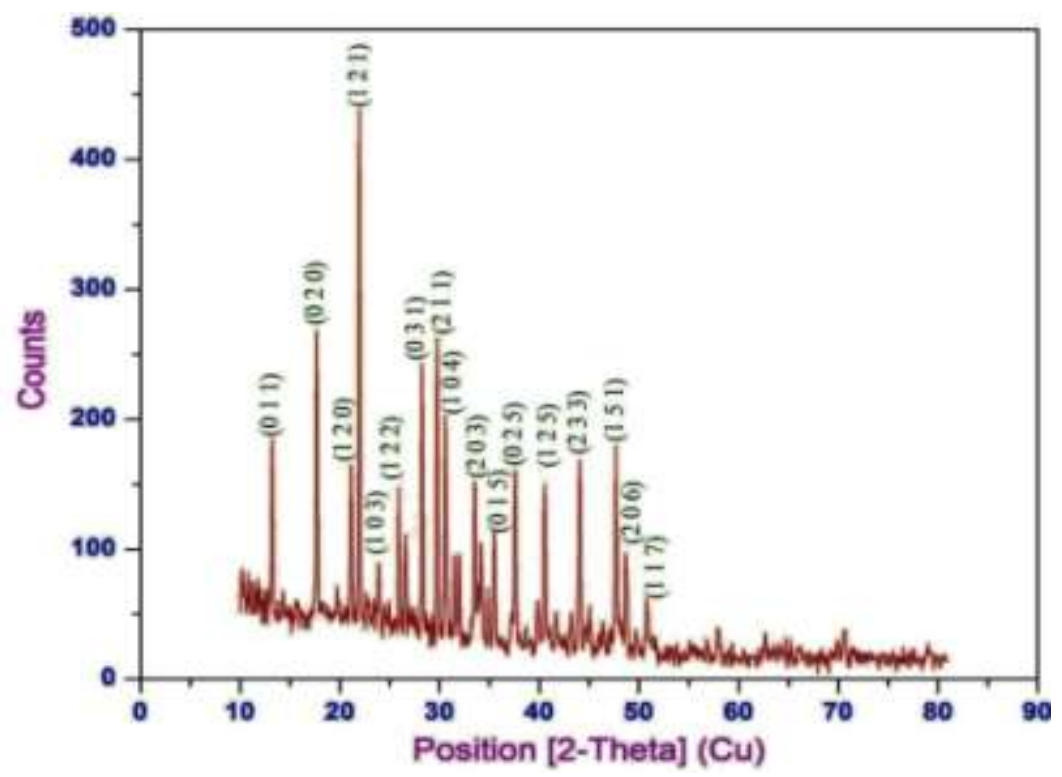

Figure 4. The powder XRD pattern of 2APKHP crystal

\subsection{FT-IR analysis}

The FT-IR spectroscopy studies are effectively used to determine the molecular structure and to identify the functional groups, presents in the grown crystal. In order to analyze qualitatively the presence of functional groups, freshly crushed powder of 2APKHP crystal was subjected to FTIR studies using thermo Nicolect v-200 FTIR spectrometer by KBr pellet method in the range $500-4000 \mathrm{~cm}^{-1}$. Figure 5 shows the FTIR spectrum of 2APKHP crystal. The absorbed frequencies and their assignment of 2APKHP crystals are shown in the table 2 . 


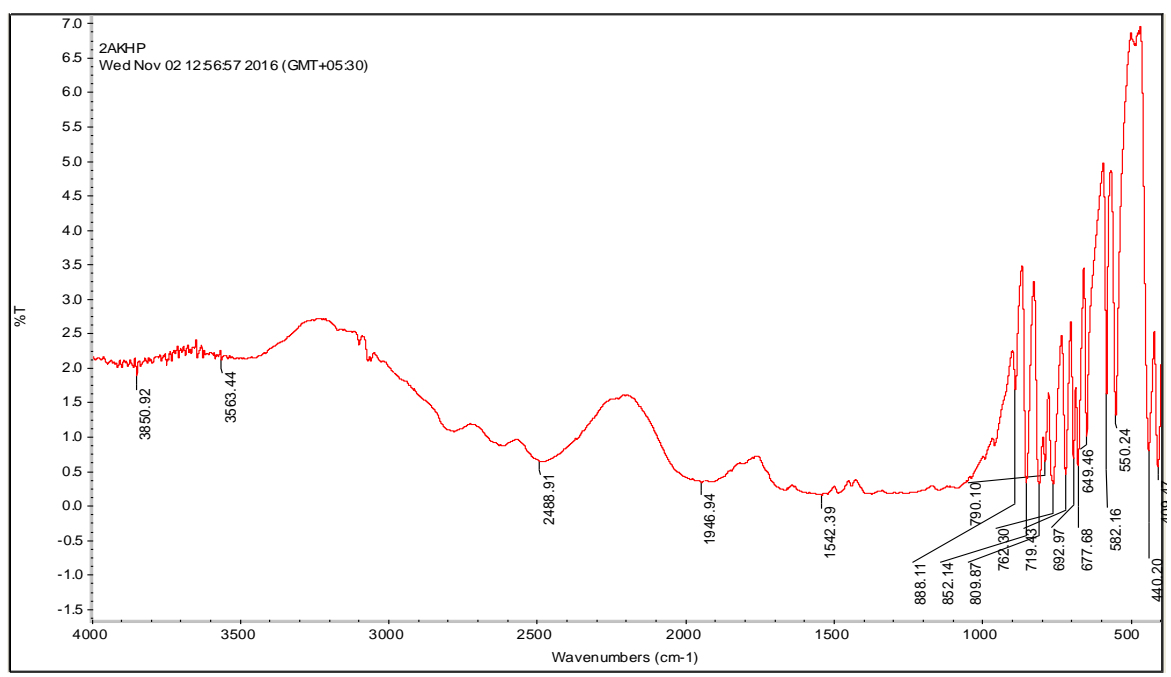

Figure 5. FT-IR spectrum of 2APKHP crystal

Table 2. Band assignments of 2APKHP crystal

\begin{tabular}{|l|l|}
\hline Wavenumber $\mathbf{~ c m}^{-1}$ & \multicolumn{1}{|c|}{ Assignments } \\
\hline 3850 & O-H stretching \\
\hline 3563 & Amide N-H stretching \\
\hline 2488 & C=O stretching \\
\hline 1946 & Aromatic overtone of ring bends \\
\hline 1542 & C-C stretching \\
\hline 888 & C-C-N symmetric stretching \\
\hline 852,809 & Out of plane C-H bending \\
\hline 762 & C-H bending \\
\hline 677 & COO- bending \\
\hline 649 & C=O wagging \\
\hline 550 & C-C-C out of plane deformation ring \\
\hline 440 & Presence of metal ion \\
\hline
\end{tabular}

\subsection{UV-vis-NIR Spectroscopy}

The optical transmission spectrum of 2APKHP crystal was recorded in the range 200-1100nm using DOUBLE BEAM UV-vis NIR spectrometer. The transmission spectrum was shown in figure 6 . The cut off wavelength is at $248 \mathrm{~nm}$. From the figure, there is no absorption band in the region $248-1100 \mathrm{~nm}$. This is an advantage of using amino acids, where the absence of strongly conjugated bonds leads to wider transparency range in the visible and UV spectral region [19].

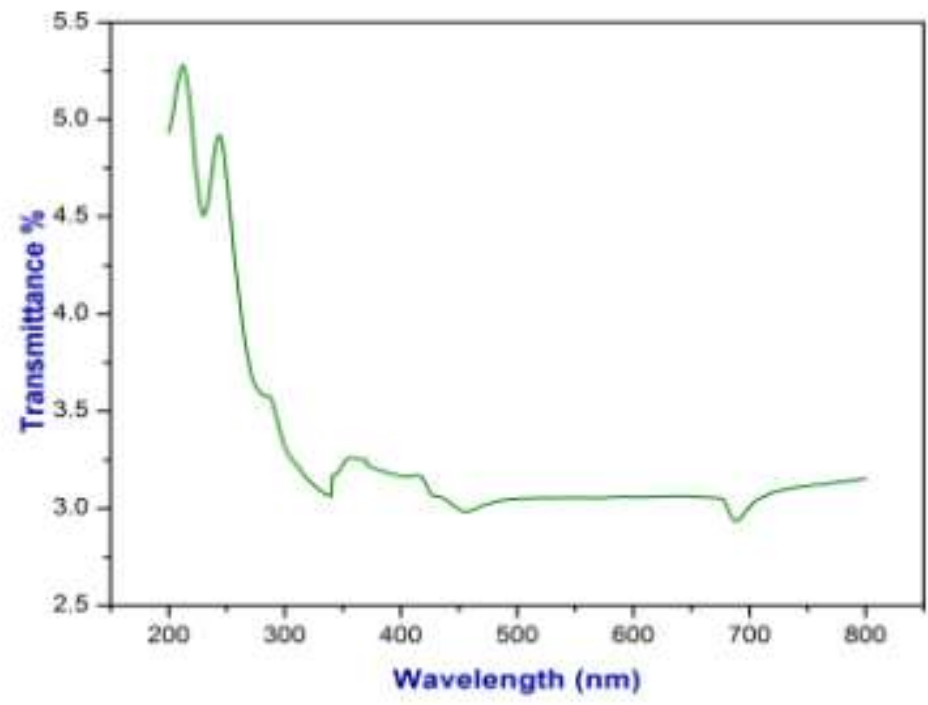

Figure 6. Optical Transmission spectrum of 2APKHP crystal 
Investigation on Synthesis, Growth, Structural and Optical Properties of a New Semiorganic ..

\subsection{Nonlinear optical study:}

The second harmonic generation (SHG) of the grown sample was confirmed by Kurtz powder technique. The powder sample of 2APKHP crystal was packed in a triangular cell and kept in a cell holder. The fundamental beam of $1064 \mathrm{~nm}$ from Q-switched Nd:YAG laser is used to test the second harmonic generation property of 2APKHP crystal by using Kurtz technique. The output from the Q-switched laser as focused into the crystal. The emission of green radiation from the 2APKHP crystal confirms the SHG in the grown crystal, which evident that the grown 2APKHP crystal is a suitable material for nonlinear optical (NLO) application.

\subsection{Dielectric studies}

The dielectric constant and the dielectric loss of the 2APKHP sample were measured using HIOKI 3532-50 LCR HITESTER. Dielectric constant and dielectric loss of the sample have been measured for different frequencies ( $100 \mathrm{~Hz}$ to $5 \mathrm{MHz}$ ) at different temperatures (308 to 348K). Figure 7 and Figure 8 show the variations of dielectric constant and dielectric loss respectively as a function of frequency at different temperatures. It is observed from Figure 7 that the dielectric constant (at $308 \mathrm{~K}$ ) decreases with increase in frequency from $100 \mathrm{~Hz}$ to $10 \mathrm{kHz}$ and then attains a constant.

The same trend is observed for other temperatures too. It is also observed that the value of dielectric constant increases with temperature. Such variations at higher temperature may be attributed to the blocking of charge carriers at the electrodes. The decrease of dielectric constant at low frequency region may be due to space charge polarization. Figure 8 indicates that as the frequency increases, the dielectric loss decreases exponentially and then attains a lower value at $308 \mathrm{~K}$. The low value of dielectric loss confirms that the sample possesses lesser defects.

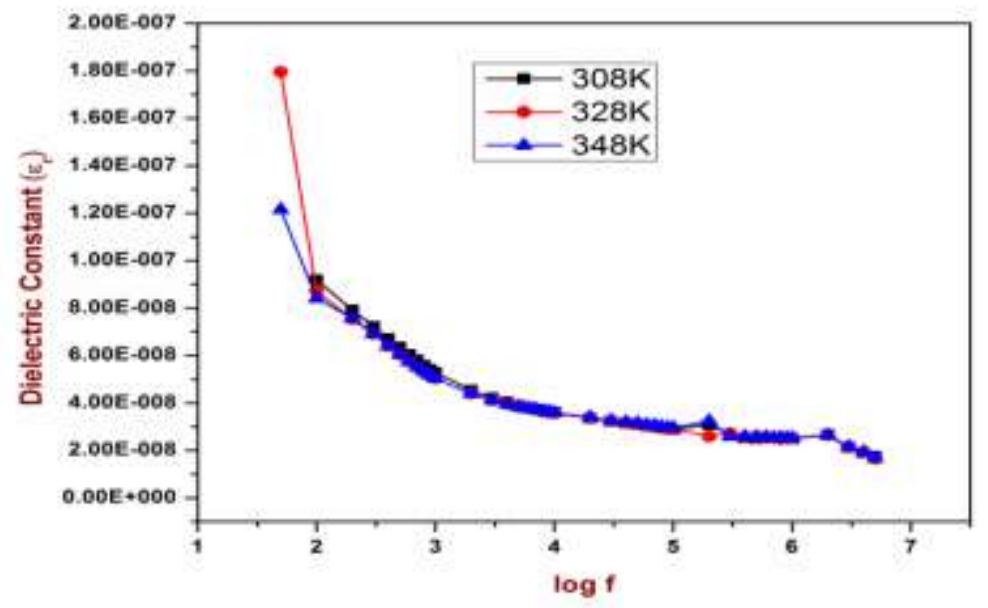

Figure 7. Variation of dielectric constant with log frequency of 2APKHP crystal

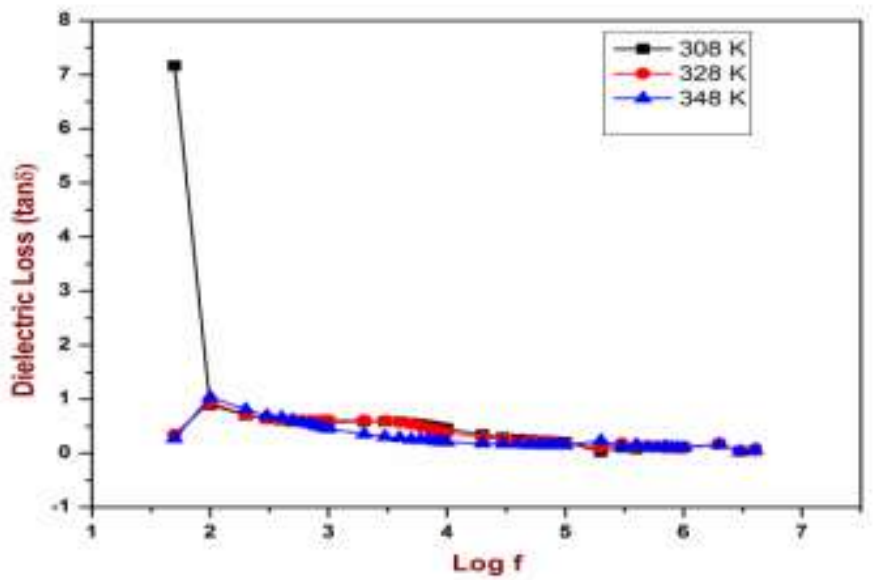

Figure 8. Variation of dielectric loss with log frequency of 2APKHP crystal 


\section{Conclusion}

A new semiorganic material 2APKHP has been synthesized and crystals were grown by slow evaporation method. The lattice parameter values have been evaluated by single crystal XRD analysis. From the XRD analysis the crystal 2APKHP belongs to orthorhombic system with space group Pca $2_{1}$. From the Powder XRD pattern, a sharp well defined peak confirmed the crystalline nature of the material. In the recorded optical transmission spectrum, the UV cut-off wavelength was found to be at $248 \mathrm{~nm}$. The SHG behavior was confirmed from the emission of bright green radiation $(532 \mathrm{~nm})$, which is evident that grown sample is a good NLO material for several applications. Dielectric constant and dielectric loss of the sample have been measured for different frequencies at different temperatures. The low value of dielectric loss confirms that the sample possesses lesser defects.

\section{Acknowledgements}

The authors are would like to thank Professor Dr. R. Jayavel, Director, Academic Research and Professor, Centre for Nanotechnology, Anna University, Chennai, for their providing facilities and also acknowledge, The Head, Dept. of Physics, B.S. Abdur Rahman University, Chennai for NLO test, The Head, Dept. of Physics, Alagappa University, Karaikkudi and SAIF, IIT-madras, Chennai.

\section{References}

[1] Santhanu Bhattacharya, Parthasarathi Dastidar, T.N. Guru Row, Chem. Mater.61 (1994)531.

[2] H.W. Zhang, A.K. Batra, R.B. Lal, J. Crystal Growth 137(1994)141.

[3] C.C. Frazier, M.P. Cockerham, J. opt. soc. Am. B 4(1987)1899.

[4] P. Hemalatha,V. Veeravazhuthi, A. Chandramohan, J. Crystal Growth 311(2009)4317-4322.

[5] N.J. Long, Angew, Chem. Int. Ed. Engl. 34(1995)21-38.

[6] D. Eimeri, S. Velsko, L. Davis, F. Wang, G. Loiacono, G. Kennedy, IEEE, J. Quantum Electron. 25(2)(1989)179-193.

[7] W.S. Wang, K. Bhat, B.G. Penn, D.O.Frazier.in; H.S. Nalwa(Ed.), Handbook of Advanced Electronic Materials and devices, vol.9, Academic press, San Diego, C A, 2001, p.193.

[8] J.R. Debord, R.C. Haushalter, J. Zubieta, J. Solid State Chem.125(1996)270-273.

[9] F. Serpaggi, G. Ferey, J. Mater. Chem. 8(1998)2737-2741.

[10] G. Xing, M. Jilang, Z. Shao, D. Xu, Chin. J. Lasers 14(1987)302-308.

[11] S. Velsko, Laser Program Annual Report, Lawrence Livermore National Laboratory, Livermore, CA, 1990.

[12] J. William(Ed). Non Linear Optical Properties of organic and polymeteric Materials, Am. Chem. Soc. Symp. Series. Vol.233, American Chemical Society, Washington, DC, 1983.

[13] D.S. Chemil, J. Zyss(Eds.), Non Linear Optical Properties of organic molecules and Crystals, Academic Press. New York, 1987(chapter1 and 2).

[14] J.F. Nicoud, R.J. Twieg, in:D.S. Chemia, J. Zyss(Eds.), Linear Optical Properties of organic molecules and Crystals, Academic Press. London, 1987. P-227.

[15] B.A. Fuchs, C. Ksyn, S.P. Velsko, Appl. Opt.28(1989)4465-4472.

[16] S.B. Monaco, L.E. Davis, S.P. Velsko, F.T. wang, D. Eimerl, A. Zalkin, J. Cryst. Growth 85(1987)252-255.

[17] R. Mohankumar, D. Rjanbabu, D. Jayaraman, R. Jayavel, K. Kitamura. J. Cryst. Growth 275(2005) 1935- 1939.

[18] X.J. Liu, Z.Y. Wang, G.H. Zhang, X.Q. Wang, A.D. Duan, Z.H. Sun et al., J. Cryst. Growth 308(2007)130-132.

[19] R. Christian, solvents and solvent effects in organic chemistry, VCH, New York, (1990).

[20] R. Srineevasan, A. Anbarasi, T. Revathi, S.M. Ravikumar, Material Chemistry and Physics 177(2016)25-30.

[21] N. Kejalakshmy, K. Srinivasan, J. Phys. D: Appl.Phys.36 (2003) 1778.

[22] A. Miniewicz, S. Bartkiewicz, Adv. Mater.opt. EWlectron.2 (1993) 157.

[23] N. Kejalakshmy, K. Srinivasan, Opt.Mater.27 (2004) 389.

[24] M Chao, E Schemp, R D Rosenstein, “2-Aminopyridine short structure paper” Acta Cryst, B31(1975) $2922-2924$. 